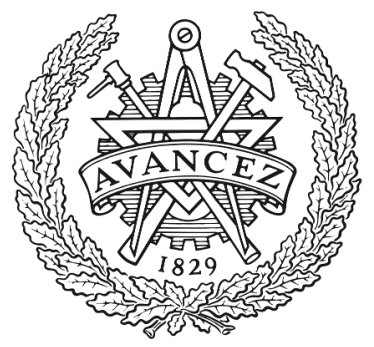

CHALMERS

UNIVERSITY OF TECHNOLOGY

\title{
Bulk-Processed Pd Nanocube-Poly(methyl methacrylate) Nanocomposites as Plasmonic Plastics for Hydrogen Sensing
}

Downloaded from: https://research.chalmers.se, 2023-04-26 14:11 UTC

Citation for the original published paper (version of record):

Darmadi, I., Stolas, A., Östergren, I. et al (2020). Bulk-Processed Pd Nanocube-Poly(methyl methacrylate) Nanocomposites as Plasmonic Plastics for Hydrogen Sensing. ACS Applied Nano Materials, 3(8): 8438-8445.

http://dx.doi.org/10.1021/acsanm.0c01907

N.B. When citing this work, cite the original published paper. 


\title{
Bulk-Processed Pd Nanocube-Poly(methyl methacrylate) Nanocomposites as Plasmonic Plastics for Hydrogen Sensing
}

\author{
Iwan Darmadi, " Alicja Stolaś,, Ida Östergren, "Barbara Berke, Ferry Anggoro Ardy Nugroho, \\ Matteo Minelli, Sarah Lerch, Irem Tanyeli, Anja Lund, Olof Andersson, Vladimir P. Zhdanov, \\ Marianne Liebi, Kasper Moth-Poulsen,* Christian Müller,* and Christoph Langhammer*
}

Cite This: ACS Appl. Nano Mater. 2020, 3, 8438-8445

Read Online

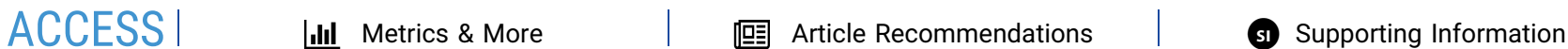

ABSTRACT: Nanoplasmonic hydrogen sensors are predicted to play a key role in safety systems of the emerging hydrogen economy. Pd nanoparticles are the active material of choice for sensor prototype development due to their ability to form a hydride at ambient conditions, which creates the optical contrast. Here, we introduce plasmonic hydrogen sensors made from a thermoplastic nanocomposite material, that is, a bulk material that can be molded with standard plastic processing techniques, such as extrusion and three-dimensional (3D) printing, while at the same time being functionalized at the nanoscale. Specifically, our plasmonic plastic is composed of hydrogensensitive and plasmonically active $\mathrm{Pd}$ nanocubes mixed with a poly(methyl methacrylate) matrix, and we optimize it by characterization from the atomic to the macroscopic level. We demonstrate melt-

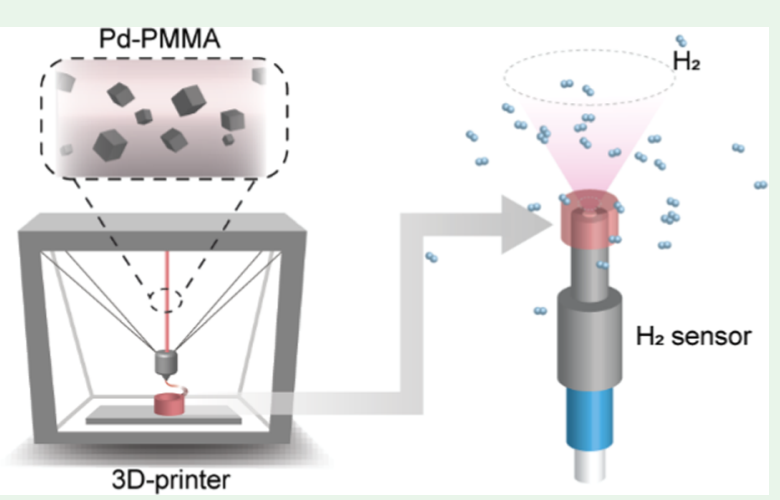
processed deactivation-resistant plasmonic hydrogen sensors, which retain full functionality even after 50 weeks. From a wider perspective, we advertise plasmonic plastic nanocomposite materials for application in a multitude of active plasmonic technologies since they provide efficient scalable processing and almost endless functional material design opportunities via tailored polymercolloidal nanocrystal combinations.

KEYWORDS: plasmonic nanocomposites, nanoparticles, polymer matrix, melt processing, 3D printing, plasmonic hydrogen sensing

\section{INTRODUCTION}

Nanoparticles supporting localized surface plasmon resonance (LSPR) enable a wide range of fascinating technologies, where optical bio- and chemosensors ${ }^{1-6}$ are of particular interest, and where hydrogen sensors show great promise as a key technology in the emerging hydrogen economy, ${ }^{6-8}$ since safety sensors are critical due to $\mathrm{H}_{2}$ 's wide flammability range in air. In this context, optical plasmonic detection is attractive since it generates no sparks due to the passive nature of the transducer and the remote readout capability of light. In this technology, the optical contrast that signals the presence of $\mathrm{H}_{2}$ in the environment stems from the absorption of hydrogen species into interstitial sites of metal nanoparticles. Therefore, plasmonic $\mathrm{H}_{2}$ sensors are intrinsically highly $\mathrm{H}_{2}$-selective. ${ }^{6} \mathrm{Pd}$ is the prototype-active $\mathrm{H}_{2}$ sensing material due to its ability to dissociate $\mathrm{H}_{2}$ gas without a sizable energy barrier at ambient conditions and its reversible phase transformation from metal to metal hydride at room temperature ${ }^{9}$ that gives rise to a large optical contrast. ${ }^{10}$ However, despite these advantages, the large-scale practical implementation of plasmonic sensors in commercial devices, in general, and of hydrogen sensors, in particular, is still lacking. A key reason for this situation is that nanofabrication approaches based on nanolithography and vacuum-based thin-film material deposition methods are mostly used for the generation of nanoplasmonic sensing functions aimed at device integration. This is both costly and limits the technology to two-dimensional (2D) arrays on flat surfaces. Thus, to enable the widespread utilization of plasmonic sensors, in general, and of hydrogen sensors, in particular, new ways to make the active plasmonic materials, as well as flexible and scalable material processing routes for three-dimensional (3D) device integration, are of critical importance. For this purpose, plasmonic nanoparticles made by colloidal synthesis ${ }^{11}$ are very attractive since they offer unrivaled possibilities for size, composition, and structural engineering to, in turn, both maximize sensitivity and optimize optical performance in plasmonic hydrogen sensors. ${ }^{12,13}$ However, even though the self-assembly of colloidal nano-

Received: July 14, 2020

Accepted: July 20, 2020

Published: July 20, 2020 


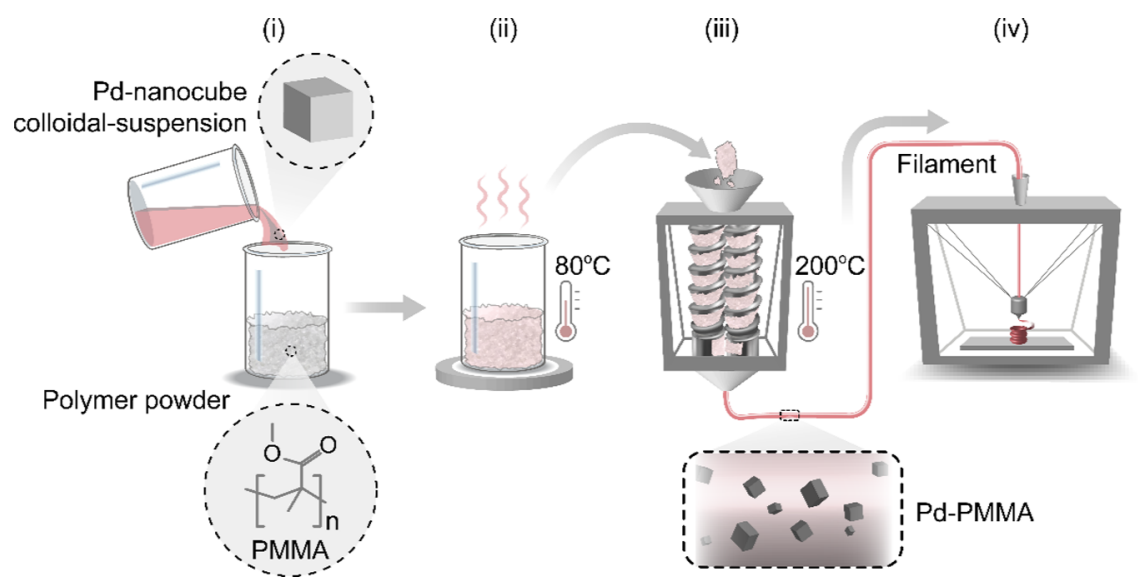

Figure 1. Plasmonic plastic Pd-PMMA nanocomposite processing scheme. Schematic depictions of mixing, compounding, and 3D printing steps (not to scale): (i) mixing of the aqueous Pd nanocube suspension with the PMMA polymer powder; (ii) drying of the PMMA-Pd nanocube mixture; (iii) melt extrusion of the composite filament; and (iv) 3D printing of a plasmonic plastic object with hydrogen sensing function.
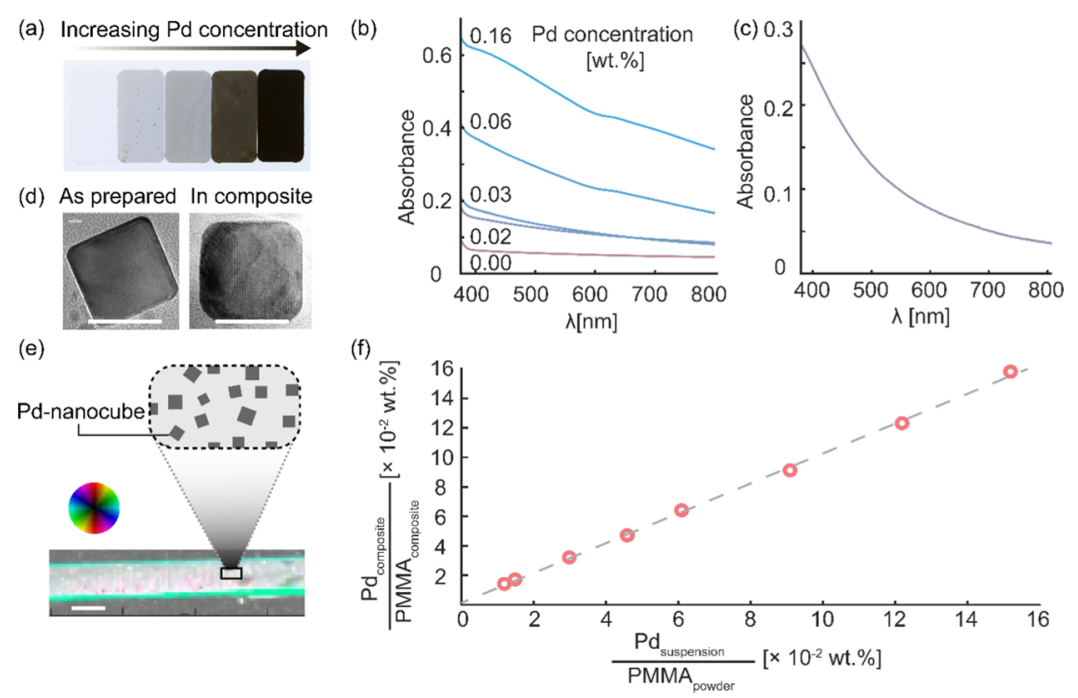

Figure 2. Pd nanocube-PMMA plasmonic plastic nanocomposite characterization. (a) Photograph of $500 \mu \mathrm{m}$ thick Pd nanocube-PMMA composite plates with Pd concentrations of $0.00,0.02,0.03,0.06$, and $0.16 \mathrm{wt} \%$. (b) Absorbance spectra of $500 \mu \mathrm{m}$ thick Pd nanocube-PMMA nanocomposite plates with the Pd loading shown in (a). (c) Absorbance spectrum of the colloidal suspension of the as-synthesized Pd nanocubes. (d) HRTEM images of a single Pd nanocube directly after colloidal synthesis and in the PMMA matrix after compounding and melt pressing. The scale bar is $20 \mathrm{~nm}$. (e) Scanning-SAXS image of a melt-pressed Pd nanocube-PMMA plate cross section with a 0.02 wt \% Pd nanocube concentration. The scale bar is $500 \mu \mathrm{m}$. The green appearance of the sample edges is due to edge scattering. (f) Pd particle concentration in synthesis suspension versus the Pd concentration in Pd nanocube-PMMA plates obtained from elemental analysis. The analysis reveals a linear correlation between the Pd particle concentration in the synthesis solution and the Pd particle concentration in the melt-pressed composite.

crystals on surfaces ${ }^{14-16}$ represents a reasonably mature approach in nanoscale processing, inherent deficiencies like nonscalability and limited adhesion call for alternative solutions for integration in sensor devices. Furthermore, hydrogen sensor deactivation due to "poisoning" by molecular species, ${ }^{17}$ such as trace amounts of CO present in, for example, urban air, ${ }^{18,19}$ or issues with cross-sensitivity ${ }^{20}$ require urgent attention.

To address these challenges, we explore here the application of an inherently scalable paradigm of melt extrusion and 3D printing of plasmonic plastic nanocomposite materials, which are composed of a polymer matrix mixed with plasmonic metal nanoparticles that provide the sensing function. This approach only requires well-established and cost-effective bulk processing techniques to shape plasmonically active objects for device integration. However, neither plasmonic plastic nanocomposite materials as such nor their processing by, e.g., melt processing, such as $3 \mathrm{D}$ printing, has so to date been considered for application in nanoplasmonic sensors in general and hydrogen sensors in particular. Therefore, as the key step beyond the state of the art, we explore here a plasmonic plastic nanocomposite material for melt-processed hydrogen sensors. As building blocks for the nanocomposite, we select colloidal $\mathrm{Pd}$ nanocubes as the active hydrogen sensing material due to their well-documented selective interaction with hydrogen via hydride formation and thus the ideal suitability as a model system to demonstrate our new concept, ${ }^{3,21,22}$ and poly(methyl methacrylate) (PMMA) as a matrix material due to its demonstrated molecular sieving function. ${ }^{7,20,23}$ After detailed structural characterization of the material to optimize its composition in terms of sensor performance, we demonstrate fully functional melt-processed plasmonic $\mathrm{H}_{2}$ gas sensors that we validate in the form of a 3D-printed sensor cap compatible with fiber optics and a melt-pressed plate integrated into a 

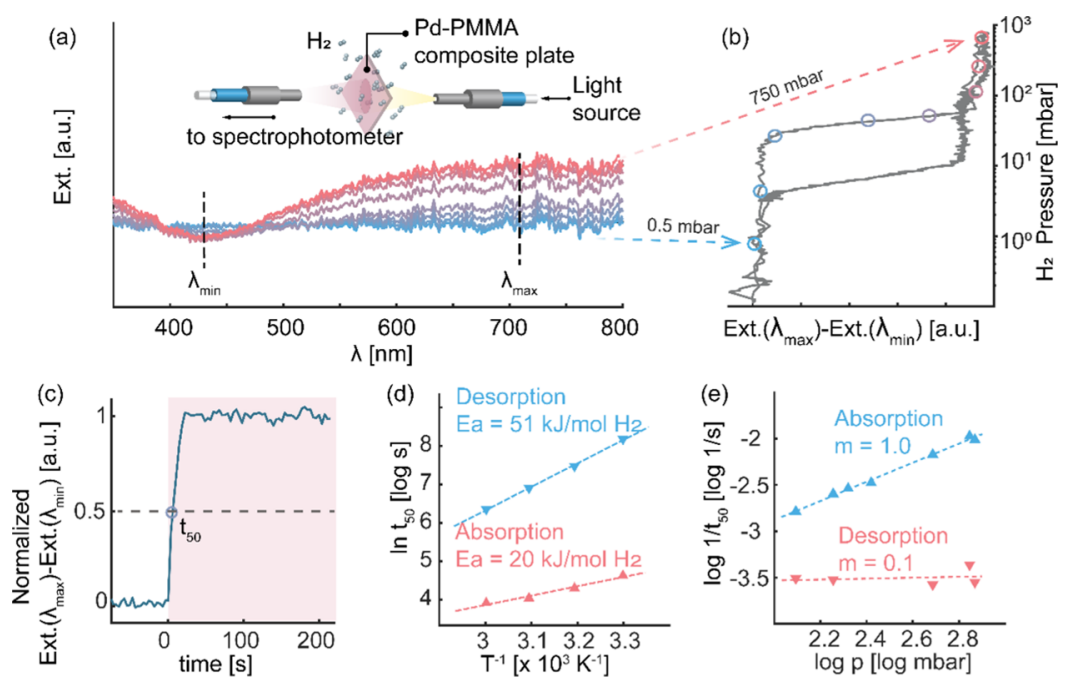

Figure 3. Pd nanocube-PMMA composite for hydrogen sensing. (a) Selected self-referenced extinction spectra evolution of a melt-pressed $50 \mu \mathrm{m}$ thick Pd nanocube-PMMA composite plate with a $0.03 \mathrm{wt} \% \mathrm{Pd}$ nanocube concentration during exposure to $0.5-750 \mathrm{mbar} \mathrm{H}_{2}$ pressure at $30{ }^{\circ} \mathrm{C}$. (b) Optical pressure-composition isotherm constructed using [extinction@ $\lambda_{\max }-$ extinction@ $\lambda_{\min }$ ] indicated in (a) as the readout parameter with the largest optical contrast. (c) Normalized temporal [extinction@ $\lambda_{\max }-$ extinction@ $\lambda_{\min }$ ] response upon the stepwise $\mathrm{H}_{2}$ pressure increase from 0 to $750 \mathrm{mbar}_{2}$ in the shaded area. (d) Arrhenius plot obtained from hydrogen ab/desorption kinetics measurements at different temperatures (Figure SI 4). The derived apparent activation energies, $E_{a}$, for hydrogen ab/desorption (solid up triangle/solid down triangle) are also shown. (e) Derivation of the sorption-order parameter, $m$, for hydrogen absorption and desorption (Figure SI 5). (c-e) were measured on a $500 \mu \mathrm{m}$ thick Pd nanocube-PMMA composite plate.

commercial prototype sensor device. Both versions readily allow optical $\mathrm{H}_{2}$ detection in air, as well as in a highly deactivating carbon monoxide ( $\mathrm{CO}$ ) background, and exhibit exceptional long-term stability of at least 50 weeks.

\section{RESULTS AND DISCUSSION}

Nanoparticle Synthesis, Composite Compounding, and 3D Printing. The first step in the process toward a functional plasmonic nanocomposite material for hydrogen sensing is the colloidal synthesis of the desired plasmonic nanocrystals. For this purpose, here, we relied on the aqueoussolution-based Pd nanocube synthesis, using hexadecyltrimethylammonium bromide (CTAB) as the stabilizing surfactant, yielding single-crystalline $\mathrm{Pd}$ nanocubes with a $23.2 \pm 3.3 \mathrm{~nm}$ average side length. In the next step, the nanoparticle dispersion was mixed with the PMMA powder and then dried for $16 \mathrm{~h}$ at elevated temperature to evaporate the aqueous solvent (Figure 1). Hence, the mixing ratio of Pd colloidal nanoparticles and polymer powder determines the colloid concentration in the final composite. Subsequently, the nanoparticle-polymer mixture was compounded in a twinscrew microcompounder and extruded into a Pd nanoparticlePMMA matrix composite filament of the desired dimension. This filament then served as the raw material for further processing of the composite into macroscopic plasmonic objects by melt pressing or 3D printing.

Pd Nanocube-PMMA Plasmonic Plastic Nanocomposite Characterization. As the active material for hydrogen detection, we have compounded Pd nanocube-PMMA composites with five different Pd nanocube loadings $(0,0.02$, $0.03,0.06$, and $0.16 \mathrm{wt} \%$ ) and melt-pressed the extruded filaments into $500 \mu \mathrm{m}$ thick plates that exhibit increasingly darker coloring for increasing nanoparticle loading (Figure 2a). The grayish appearance is in good agreement with an LSPR resonance peak in the UV spectral range and the broad-band spectral absorbance of Pd nanoparticles due to interband damping of the LSPR ${ }^{24,25}$ both in the composite (Figure $2 \mathrm{~b}$ ) and in a colloidal suspension (Figure 2c). Furthermore, the inspection of high-resolution transmission electron microscopy (HRTEM) images of the Pd nanocubes before (in colloidal suspension) and after compounding (inside the polymer matrix) reveals the general cubic shape preservation (with an indication of slight edge-rounding) and their single-crystalline nature (Figures $2 \mathrm{~d}$ and SI 1). Scanning-SAXS analysis of the composite with a $0.02 \mathrm{wt} \% \mathrm{Pd}$ nanocube concentration in the q-range of $0.006-0.55 \AA^{-1}$ shows a homogeneous distribution and isotropic arrangement throughout the cross-sectional slice (Figure 2e, minor segregation is observed; see Figure SI 2a-c), and also corroborates the anticipated average size of about 28.0 $\pm 1.8 \mathrm{~nm}$ of the Pd nanocubes in the composite (Figure SI $2 \mathrm{~d}$ and Table SI 1). Finally, elemental analysis reveals that the weight ratio of the Pd nanocubes in the synthesis suspension to the PMMA powder scales linearly with the weight ratio of the Pd to the PMMA in the Pd nanocube-PMMA nanocomposite (Figure 2f). Moreover, the elemental analysis also implies that no Pd is lost during the processing.

Pd Nanocube-PMMA Nanocomposites for Plasmonic Hydrogen Detection. For the first global characterization of the Pd nanocube-PMMA nanocomposite interaction with $\mathrm{H}_{2}$ and its corresponding optical response, we melt-pressed a $50 \mu \mathrm{m}$ thick plate with a $0.03 \mathrm{wt} \% \mathrm{Pd}$ nanocube loading. Using a vacuum chamber setup with optical windows, ${ }^{26}$ we then measured the wavelength-resolved selfreferenced (with respect to the first measured spectrum of the experiment) optical extinction of the composite for increasing $\mathrm{H}_{2}$ gas pressure (Figure $3 \mathrm{a}$ ). We focus on the wavelength range corresponding to the flank of the plasmonic "peak", which has been shown to yield the highest sensitivity. ${ }^{26,27}$ The selected spectra reveal the corresponding distinct optical contrast of the composite. Continuous monitoring of the extinction difference at two selected wavelengths, $\lambda_{\min }$ and $\lambda_{\max }$, thus enables the optical mapping of a pressure-composition isotherm of the 
material due to the linear dependence of the Pd plasmonic response to $\mathrm{H}_{2}{ }^{10,28,29}$ (Figure $3 \mathrm{~b}$ ). The obtained isotherm is in excellent agreement with other studies of $\mathrm{Pd}$ nanocube $-\mathrm{H}_{2}$ interactions and exhibits the characteristic low-pressure $\alpha$ phase, the $\alpha+\beta$-phase coexistence plateau, and the highpressure $\beta$-phase regions, as well as distinct hysteresis between absorption and desorption in the two-phase region. ${ }^{3,21,22}$ This corroborates that the intrinsic material properties of the $\mathrm{Pd}$ nanocrystals are preserved in the nanocomposite, guaranteeing the anticipated functionality (for the corresponding analysis of neat Pd nanocubes, see Figure SI 3a,b). At the same time, this Pd-specific characteristic response to $\mathrm{H}_{2}$ exposure has implications for sensor performance. As apparent from the measured isotherm, the most significant optical contrast change occurs at the $\alpha+\beta$-phase coexistence plateau, while it is very small in the $\alpha$-phase region. This means that the material in its current form, and as characteristic for most $\mathrm{H}_{2}$ sensors based on pure $\mathrm{Pd}$, is most useful when applied for the detection of a critical $\mathrm{H}_{2}$ threshold pressure corresponding to the two-phase coexistence plateau. To resolve this limitation and thereby significantly improve sensor detection limit, as well as to eliminate hysteresis, alloying of Pd with, e.g., $\mathrm{Au}^{26}$ or $\mathrm{Cu}^{17}$ has proven very effective, and we thus propose this approach also for next-generation plasmonic plastics-based hydrogen sensors.

To quantify the kinetics of hydrogen sorption in the $\mathrm{Pd}$ nanocube-PMMA composite, we measured the temporal response to stepwise $\mathrm{H}_{2}$ exposure and used $t_{50}$, that is, the response time to $50 \%$ of the maximal steady-state signal at the new pressure (Figure 3c), as the descriptor. As the corresponding Arrhenius analysis reveals, we find apparent activation energies of 20 and $51 \mathrm{~kJ} / \mathrm{mol} \mathrm{H}_{2}$ for hydrogen absorption and desorption in the composite, respectively (Figures 3d and SI 4, and Section SI 5), which is within the range of reported literature values for the $\mathrm{Pd}-\mathrm{H}$ system. ${ }^{7,30}$ Furthermore, analysis of the pressure dependence yields an order parameter, $m=1$, for absorption and $m=0.1$ for desorption (Figures $3 \mathrm{e}$ and SI 5), indicating pressure independence in the latter case.

Having established a fundamental mechanistic understanding of the $\mathrm{Pd}$ nanocube-PMMA nanocomposite $-\mathrm{H}_{2}$ interaction, it is now interesting to investigate the impact of nanocomposite design parameters on $\mathrm{H}_{2}$ sensing performance. We prepared a series of melt-pressed plates with (i) different Pd nanocube loadings (0.02-0.16 wt \%) at constant $250 \mu \mathrm{m}$ thickness and (ii) different thicknesses $(100-800 \mu \mathrm{m})$ at a constant 0.02 wt \% Pd nanocube concentration. Subsequently, we measured their response to a $750 \mathrm{mbar}_{2}$ pressure step (see Figures 4a,c and SI 6 and 7 for recovery). Plotting $t_{50}$ vs nanocube concentration (Figure $4 \mathrm{~b}$ ) and vs plate thickness (Figure 4d) reveals distinct linear and quadratic dependencies, respectively. To understand these, we developed an analytical model based on propagation of a $\mathrm{H}_{2}$ diffusion front through the composite (Figure 4e and Subsection SI 7.1). Our model suggests that the transient kinetics of hydrogen sorption in the composite depends on the balance between $\mathrm{H}_{2}$ diffusion in the PMMA matrix and $\mathrm{H}$ sorption by the Pd nanocubes. Hence, we predict that the absorption time scales linearly with nanoparticle loading and shows a quadratic dependency on the plate thickness, and fit $t_{50}=k_{\mathrm{a}}^{\text {conc }} C_{\mathrm{NP}}\left(\right.$ eq S16) and $t_{50}=k_{\mathrm{a}}^{\text {thick }} L^{2}$ (eq S18) to the experimental data in Figure 4b,d, respectively. $C_{\mathrm{NP}}$ and $L$ are the nanocube concentration and plate thickness, respectively. The fitting enables the extraction of $k_{\mathrm{a}}^{\text {conc }}$ and (a)
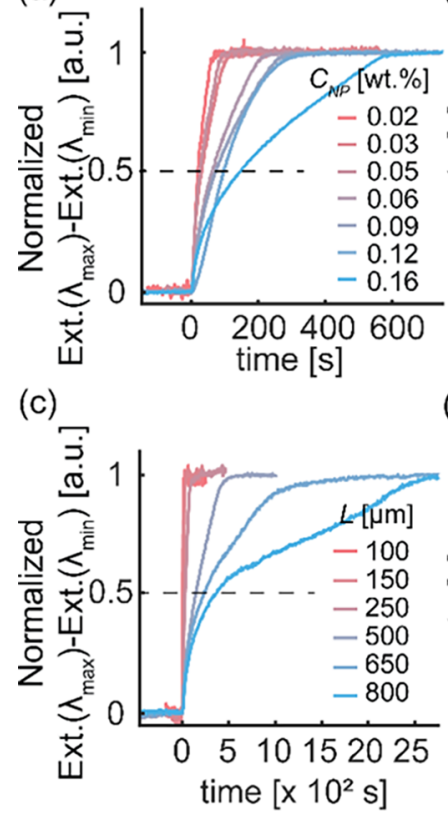

(b)

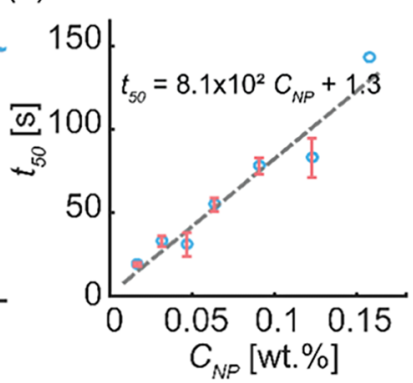

(d)

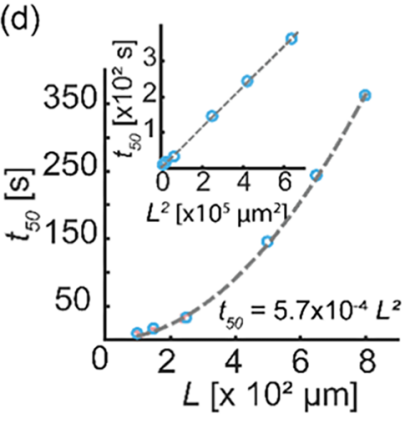

(e)

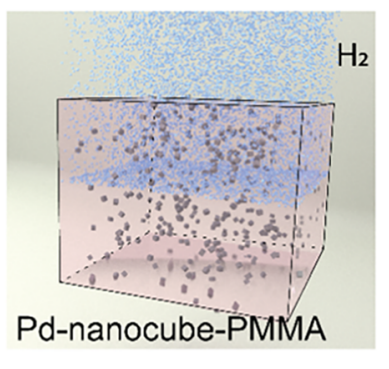

(f) $\quad C_{N P}[w t . \%]$

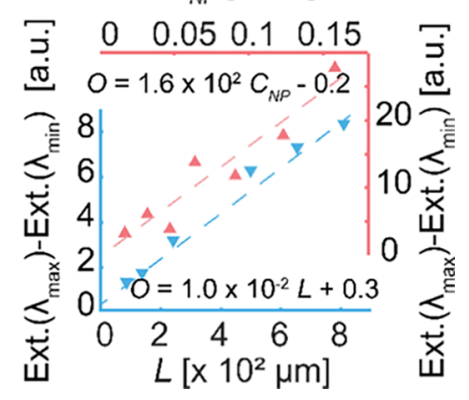

Figure 4. Hydrogen sensing kinetics and optical contrast of $\mathrm{Pd}$ nanocube-PMMA. (a) Normalized optical response to a stepwise $\mathrm{H}_{2}$ pressure increase from 0 to $750 \mathrm{mbar}$ at $30^{\circ} \mathrm{C}$ for melt-pressed $\mathrm{Pd}$ nanocube-PMMA plates with different Pd loadings. (b) Temporal response $\left(t_{50}\right)$ for the samples presented in (a) as a function of the Pd nanocube concentration in the composite. Error bars depict the standard deviation from three measurements for each sample. (c) Normalized optical response of melt-pressed Pd nanocube-PMMA plates with different thicknesses at a constant $\mathrm{Pd}$ nanocube concentration of $0.02 \mathrm{wt} \%$. (d) Temporal response $\left(t_{50}\right)$ for the samples presented in (c), plotted as a function of plate thickness, $L$, revealing a quadratic trend. The inset shows the same data plotted vs $L^{2}$. (e) Schematic mechanistic depiction of the analytical model used to analyze the experimental data. It assumes the propagation of a $\mathrm{H}_{2}$ diffusion front. (f) Optical contrast $(\mathrm{O})$ upon hydrogenation of the nanocomposite to $750 \mathrm{mbar} \mathrm{H}_{2}$, defined as [extinction@ $@ \lambda_{\max }-$ extinction@ $\left.\lambda_{\min }\right]$, for plates with different Pd nanocube concentrations (solid up triangle) and different thicknesses at a constant $\mathrm{Pd}$ nanocube concentration (solid down triangle). The dashed lines correspond to linear fits to the data.

$k_{\mathrm{a}}^{\text {thick}}$, which are related to the $\mathrm{H}_{2}$ diffusion constant in PMMA as

$$
k_{\mathrm{a}}^{\text {conc }}=c_{0} V_{\mathrm{NP}} L^{2} /\left[64\left(c_{\mathrm{f}}-\hat{c}_{\mathrm{f}}^{*}\right) D_{\text {conc }}\right]
$$

for varying Pd nanoparticle concentration, and

$$
k_{\mathrm{a}}^{\text {thick }}=c_{0} C_{\mathrm{NP}} V_{\mathrm{NP}} /\left[64\left(c_{\mathrm{f}}-\hat{c}_{\mathrm{f}}^{*}\right) D_{\text {thick }}\right]
$$

for varying plate thickness. Here, $c_{0}$ is the $\mathrm{H}$ concentration in the hydride, $V_{\mathrm{NP}}$ is the nanocube volume, $c_{\mathrm{f}}$ is the $\mathrm{H}_{2}$ 
concentration in the PMMA matrix, and $\hat{c}_{\mathrm{f}}^{*}$ is the $\mathrm{H}_{2}$ concentration in the PMMA matrix when the nanocubes are fully hydrated. $\hat{c}_{\mathrm{f}}^{*}$ is thus proportional to the absorption plateau pressure of $\mathrm{Pd}$. The obtained diffusion constants are $D_{\text {conc. }}=$ $4.4 \times 10^{-7} \mathrm{~cm}^{2} / \mathrm{s}$ and $D_{\text {thick }}=1.6 \times 10^{-7} \mathrm{~cm}^{2} / \mathrm{s}$. These values are in good agreement with the corresponding value $D_{\text {diff }}=6.3$ $\times 10^{-7} \mathrm{~cm}^{2} / \mathrm{s}$ obtained from direct diffusivity measurements (Figure SI 8 and Table SI 3). This means that the predominant rate-limiting factor for $\mathrm{H}_{2}$ absorption in the nanocomposite is $\mathrm{H}_{2}$ diffusion through the PMMA matrix and not $\mathrm{H}_{2}$ sorption by the Pd nanocubes, in agreement with the faster response of neat Pd nanocubes (Figure SI 3c). Finally, we also quantified the optical contrast, defined as [extinction@ $\lambda_{\max }-$ extinction@ $\lambda_{\text {min }}$ ], between the composite in the unhydrogenated and hydrogenated states at a $750 \mathrm{mbar}_{2} \mathrm{H}_{2}$ pressure (Figure 4f). Evidently, the measured optical contrast scales linearly both with increasing plate thickness at a constant Pd nanocube loading and with increasing loading at a constant thickness, in good agreement with the Beer-Lambert law.

In summary, these observations imply a trade-off between the optical contrast and temporal response for sensing. Hence, there is an optimal Pd loading and plate thickness that provides the best compromise between those two figures of merit, and we argue that it is preferable to enhance the sensing contrast by increasing the Pd loading rather than the plate thickness because the former increases the temporal response linearly, while the latter shows a quadratic dependency. Also, we predict that further tailoring of the polymer matrix in terms of $\mathrm{H}_{2}$ permeability is a viable strategy to significantly improve the temporal response of such systems in the future.

Melt-Pressed and 3D-Printed Deactivation-Resistant Plasmonic Plastic Hydrogen Sensors. To demonstrate the ease of integration of our plasmonic plastic into real devices, we tested two different systems as deactivation-resistant $\mathrm{H}_{2}$ sensors in the CO-rich environment: ${ }^{31}$ (i) a melt-pressed Pd nanocube-PMMA sensor plate of $100 \mu \mathrm{m}$ thickness with a Pd nanocube loading of 0.03 wt $\%$ and (ii) a 3D-printed $\mathrm{Pd}$ nanocube-PMMA sensor cap of a $200 \mu \mathrm{m}$ effective thickness and with a 0.02 wt \% Pd nanocube loading. The $200 \mu \mathrm{m}$ thickness of the 3D-printed cap is dictated by the nozzle of the used $3 \mathrm{D}$ printer. To compensate for the increased thickness and its detrimental impact on response time, we have therefore reduced the $\mathrm{Pd}$ loading slightly, according to the design rule introduced above.

For the first test, we inserted the melt-pressed Pd nanocube-PMMA plate into a gas sensor device (Insplorion $\mathrm{AB}$, Göteborg, Sweden; Figure 5a). We then exposed the sensor to subsequent cycles of $10 \% \mathrm{H}_{2}$, of $0.5 \% \mathrm{CO}$, and of $10 \% \mathrm{H}_{2}+0.5 \% \mathrm{CO}$, all in the synthetic air carrier gas. Remarkably, thanks to the low diffusivity of CO in PMMA, ${ }^{31}$ the system exhibits consistent and reliable response to $\mathrm{H}_{2}$ even at severe $\mathrm{CO}$ poisoning conditions, which would readily deactivate the unprotected $\mathrm{Pd}$ control via strong $\mathrm{CO}$ chemisorption and the concurrent blocking of $\mathrm{H}_{2}$ dissociation sites (Figure $5 \mathrm{~b}) .^{18}$

For the second test, we opted for a fiber-optic sensor solution onto which we fitted the tailor-made $3 \mathrm{D}$-printed sensor cap (Figure 5c). For sensing performance testing, the cap was mounted on the tip of an SMA-terminated optical fiber mounted inside a homebuilt flow reactor using a fiberoptic feed-through (Figure SI 10c) and then exposed to subsequent cycles of $10 \% \mathrm{H}_{2}$, of $0.5 \% \mathrm{CO}$, and of $10 \% \mathrm{H}_{2}+$ $0.5 \% \mathrm{CO}$, all in the synthetic air carrier gas. Similar to the melt- (a)

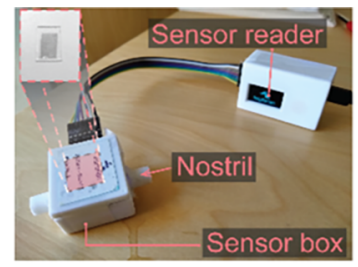

(b)

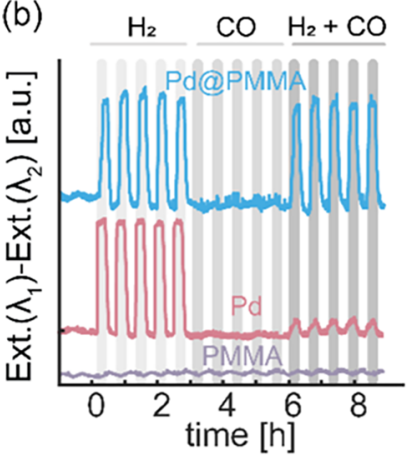

(c)

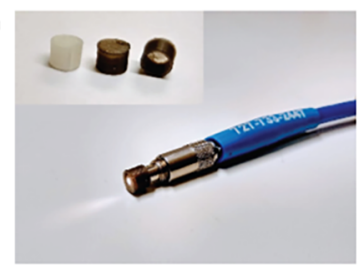

(d)

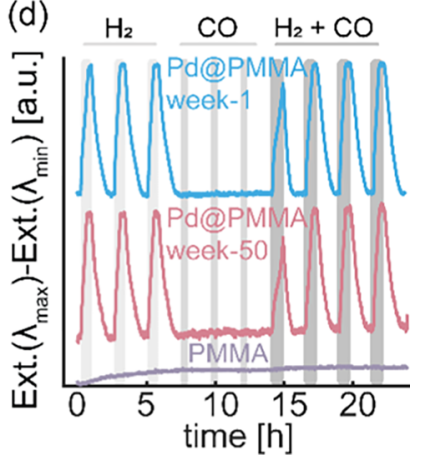

Figure 5. Device integration of melt-pressed and 3D-printed $\mathrm{Pd}$ nanocube-PMMA $\mathrm{H}_{2}$ sensors. (a) Photograph of the plasmonic gas sensor prototype device provided by Insplorion $\mathrm{AB}$. The inset depicts a Pd nanocube-PMMA sensor plate with a Pd nanocube loading of $0.03 \mathrm{wt} \%$, dimensions of $6 \mathrm{~mm} \times 12 \mathrm{~mm}$ and a thickness of $100 \mu \mathrm{m}$. (b) Sensor response expressed as [extinction@ $\lambda_{1}-$ extinction@ $\lambda_{2}$ ] for the Pd nanocube-PMMA nanocomposite, for neat Pd nanocubes drop-casted onto the fused silica support, and a melt-pressed neat PMMA control. The samples were exposed to five subsequent cycles of $10 \% \mathrm{H}_{2}$, five cycles of $0.5 \% \mathrm{CO}$, and five cycles of $10 \% \mathrm{H}_{2}+0.5 \%$ $\mathrm{CO}$, all in the synthetic air carrier gas. (c) Photograph of a 3D-printed Pd nanocube-PMMA composite sensor cap with a 0.02 wt \% Pd loading plugged onto an SMA-905 multimode optical fiber. The nanocomposite material thickness at the tip of the cap is $200 \mu \mathrm{m}$. Inset: photograph of 3D-printed neat PMMA (white) and $\mathrm{Pd}$ nanocube-PMMA sensor caps (dark gray). (d) Fresh and 50 weeks aged 3D-printed Pd nanocube-PMMA and neat PMMA sensor cap response, defined as [extinction@ $\lambda_{\max }-$ extinction@ $\lambda_{\min }$ ], to three subsequent cycles of $10 \% \mathrm{H}_{2}$, three cycles of $0.5 \% \mathrm{CO}$, and four cycles of $10 \% \mathrm{H}_{2}+0.5 \% \mathrm{CO}$, all in the synthetic air carrier gas.

pressed sensor plate, the 3D-printed fiber cap sensor remained fully responsive even at severe $\mathrm{CO}$ poisoning conditions, thereby confirming the anticipated selective molecular sieving function of the PMMA matrix and the selective response of the $\mathrm{Pd}$ nanocubes to $\mathrm{H}_{2}$ (Figure 5d, blue curve). ${ }^{31}$ The 3D-printed neat PMMA control also in this case exhibited no response to any of the tested gases (Figure $5 \mathrm{~d}$, gray curve).

Finally, to test the long-term stability of our plasmonic plastic nanocomposite, we repeated the same experiment after aging of the 3D-printed sensor cap for 50 weeks at ambient conditions in air. Remarkably, the response to hydrogen, as well as the protection against deactivation by $\mathrm{CO}$, is virtually unchanged (Figure 5d, red curve). This result thus advertises plasmonic plastic materials as an attractive solution for hydrogen sensors where frequent sensor recalibration due to aging and deactivation is one of the main limitations of existing commercial sensor solutions.

\section{CONCLUSIONS}

Plasmonic plastic nanocomposites provide a flexible material platform for melt-processed active plasmonic sensor devices, where functions like molecular sieving and ease of economic and scalable processing are intrinsically included. Furthermore, 
the melt-processed plasmonic plastic nanocomposite- $\mathrm{H}_{2}$ gas sensors we have presented challenge existing plasmonic $\mathrm{H}_{2}$ sensor platforms, utilizing $2 \mathrm{D}$ arrays of nanofabricated or selfassembled nanoparticles on flat surfaces, since they are a conceptually fundamentally different paradigm for the scalable production of $3 \mathrm{D}$ devices. In our plasmonic plastic nanocomposite material, the plasmonically active and hydrideforming $\mathrm{Pd}$ nanocubes respond selectively to $\mathrm{H}_{2}$ through a distinct change in their optical properties. At the same time, the PMMA matrix: (i) acts as a molecular sieve that prevents sensor deactivation by other molecular species, such as $\mathrm{CO}$; (ii) is a means to stabilize the shape of the embedded nanocrystals; (iii) prevents their aggregation; and (iv) ensures sensor long-term stability, thereby drastically reducing the need for recalibration. This breakthrough was corroborated by the successful testing of melt-pressed and 3D-printed sensor plates and caps, respectively, that were directly integrated with a miniaturized optical sensor device prototype and with an offthe-shelf fiber-optic solution. These implementations were then successfully used to demonstrate $\mathrm{H}_{2}$ detection in simulated application conditions in synthetic air with deactivating $\mathrm{CO}$ contamination, and revealed virtually unchanged sensor response and deactivation resistance even after 50 weeks of plasmonic plastic sensor aging at ambient conditions in air.

In a wider perspective, our work enables next-generation optical gas sensors centered on the idea of melt-processed plasmonic plastic nanocomposite materials that combine tailored plasmonic nanocrystal signal transducers with polymer matrix materials, selected and optimized based on the specific analyte to be detected. Furthermore, we envision the application of these materials in other branches of plasmonics, including magnetoplasmonics, plasmonic metamaterials, and plasmon-enhanced photovoltaics, where cost-effective scalable fabrication is critical for large-scale commercial integration in real devices and where the almost endless opportunities provided by combining polymer and colloidal chemistry will enable compelling opportunities in terms of rational material design and function.

\section{METHODS}

Nanoparticle Synthesis. The following chemicals were used: hexadecyltrimethylammonium bromide (CTAB, $\geq 98.0 \%$ SigmaAldrich), L-ascorbic acid (99\% Sigma-Aldrich), sodium borohydride $(\geq 98.0 \%)$, palladium(II) chloride (99\% Sigma-Aldrich), and hydrochloric acid $37 \%$ (Sigma-Aldrich).

Pd nanocubes stabilized with CTAB were synthesized using a procedure reported elsewhere ${ }^{32}$ with slight modifications where appropriate. A $10 \mathrm{mM} \mathrm{H}_{2} \mathrm{PdCl}_{4}$ solution was prepared by dissolving $0.1773 \mathrm{~g} \mathrm{PdCl}_{2}$ in $10 \mathrm{~mL}$ of $0.2 \mathrm{M} \mathrm{HCl}$; thereafter, the solution was diluted up to $100 \mathrm{~mL}$ in Milli-Q $\mathrm{H}_{2} \mathrm{O}(18.2 \mathrm{M} \Omega) . \mathrm{H}_{2} \mathrm{PdCl}_{4}(0.5 \mathrm{~mL})$ was added to a $12.5 \mathrm{mM} \mathrm{CTAB}$ solution. The mixture was heated up to $95^{\circ} \mathrm{C}$, and $80 \mu \mathrm{L}$ of $100 \mathrm{mM}$ ascorbic acid was added under rapid stirring. Subsequently, the mixture was kept in the water bath for 30 $\mathrm{min}$. Next, the reaction mixture was centrifuged at $8000 \mathrm{rpm}$, and the obtained pellet was dispersed in Milli- $\mathrm{Q} \mathrm{H}_{2} \mathrm{O}$ to obtain $4 \times 10^{11}$ $[\mathrm{NPs} / \mathrm{mL}]$. To test the role of nanoparticle concentration in the nanocomposite in $\mathrm{H}_{2}$ sensing, $\mathrm{Pd}$ nanocubes were also synthesized with the following concentrations: $1 \times 10^{11}, 5 \times 10^{11}, 1 \times 10^{12}, 2 \times$ $10^{12}$, and $5 \times 10^{12}[\mathrm{NPs} / \mathrm{mL}]$ by changing the volumes while maintaining the ratio and concentrations of the reactants.

Composite Compounding. Poly(methyl methacrylate), PMMA, powder with a weight-average molecular weight, $M_{w}$, of $75000 \mathrm{~g} / \mathrm{mol}$, a polydispersity index, PDI, of 2.8 and a density of $1.2 \mathrm{~g} / \mathrm{cm}^{3}$ was obtained from Polyscience Inc. The Pd-PMMA composite was prepared by pouring the aqueous $\mathrm{Pd}$ nanoparticle dispersions onto the PMMA powder, followed by drying at $80{ }^{\circ} \mathrm{C}$ overnight. The dry mixtures were compounded for $5 \mathrm{~min}$ in an Xplore Microcompounder MC5 at $200{ }^{\circ} \mathrm{C}$, extruded through a $1.0 \mathrm{~mm}$ die, and collected onto a conveyor belt at slow speed, resulting in filaments with a diameter of $1.75 \pm 0.1 \mathrm{~mm}$.

Melt Pressing of Plates. Melt-pressed plates were produced with a hot press from $A B$ Nike Hydralik, using extruded filaments as raw material. The Pd nanocube-PMMA samples were pressed at $200{ }^{\circ} \mathrm{C}$ and 5 tons for $3 \mathrm{~min}$ and then under 10 tons for $2 \mathrm{~min}$, with metal spacers to control their final thickness ranging from 50 to $800 \mu \mathrm{m}$. For $\mathrm{H}_{2}$ sensing with the prototype sensor device, $100 \mu \mathrm{m}$ thick plates were melted onto a fused silica substrate for structural support.

3D Printing via Fused Filament Fabrication. For the fused filament fabrication of the Pd-PMMA composite, we used a Massportal Pharaoh XD printer. The printing was carried out at a nozzle temperature of $210^{\circ} \mathrm{C}$, with a build-plate temperature of $60^{\circ} \mathrm{C}$ and a printing speed of $1000 \mathrm{~mm} / \mathrm{min}$.

Elemental Analysis. Elemental analysis for the Pd content in the composite was performed on samples dried at $60{ }^{\circ} \mathrm{C}$ for $4 \mathrm{~h}$ at MIKROLAB, Kolbe Germany.

Transmission Electron Microscopy. All nanoparticle and nanocomposite samples were imaged using a Tecnai T20 TEM microscope with a LaB6 gun, operating at $200 \mathrm{kV}$ or a Titan $80-300$ TEM microscope with a field emission gun, operating at $300 \mathrm{kV}$. The nanoparticle solution sample was prepared on a pure carbon 200 mesh copper grid, and Pd-PMMA nanocomposites were prepared on a Lacey 400 mesh copper grid.

UV-Vis Spectroscopy. The UV-visible absorbance spectra were obtained with a Cary 5000 spectrophotometer within the 350-800 $\mathrm{nm}$ spectral range.

Scanning Small-Angle X-ray Scattering (Scanning-SAXS). Scanning-SAXS measurements were carried out at the cSAXS beamline of the Swiss Light Source (SLS) at the Paul Scherrer Institute (PSI) in Villigen, Switzerland. The sample was scanned at an energy of $11.2 \mathrm{keV}$, and the beam size was $40 \mu \mathrm{m} \times 40 \mu \mathrm{m}$. The sample-to-detector distance was $2.16 \mathrm{~m}$. A Pilatus $2 \mathrm{M}$ detector was used to acquire small-angle scattering patterns. The step sizes used were $40 \mu \mathrm{m} \times 40 \mu \mathrm{m}$, while the applied exposure time was $0.2 \mathrm{~s}$. Data processing was carried out using the "cSAXS scanning SAXS package" developed by the CXS group, Paul Scherrer Institute, Switzerland. ${ }^{33}$ The size determination was performed using the SASView 4.2.0 software.

Hydrogen Sensing Experiments. The $\mathrm{H}_{2}$ sensing experiments were carried out in (i) a vacuum chamber described in detail elsewhere $^{7,26}$ (Figure SI 9), (ii) a homebuilt flow reactor (Figure SI 10), and (iii) the hydrogen sensor prototype device. The measurements in the vacuum chamber and in the flow reactor were executed at $30{ }^{\circ} \mathrm{C}$ (the temperature was maintained by external heating), while those in the homebuilt flow reactor and the prototype sensor device were done at $21^{\circ} \mathrm{C}$ (ambient temperature of the testing lab). We used multimode optical fibers and a spectrophotometer (Avantes SensLine AvaSpec-2048XL). In the flow reactor, the gas flow was regulated by mass flow controllers (Bronkhorst Low $\Delta P$ ). The total gas flow was $200 \mathrm{~mL} / \mathrm{min}$ using synthetic air as the carrier gas. The 3D-printed fiber caps were mounted in a flow reactor equipped with a CF flange fiber-optic feed-through and a silica vacuum viewport connected to a light source (Avantes AvaLight-Hal Mini) and the spectrophotometer, respectively. The sensing was performed in transmission mode. The prototype sensor device is equipped with both an internal light source and a detector and operates in transmission mode. It was connected to the same set of mass flow controllers for the gas flow and composition regulation as the flow reactor.

\section{ASSOCIATED CONTENT}

\section{Supporting Information}

The Supporting Information is available free of charge at https://pubs.acs.org/doi/10.1021/acsanm.0c01907. 
Transmission electron microscopy of Pd nanocubes after synthesis and compounding; small-angle X-ray scattering for Pd nanocube-PMMA; Pd nanocube isotherm and response/recovery to/from a 750 mbar hydrogen pressure; raw data for the Arrhenius analysis of hydrogen sorption kinetics; calculation of the apparent activation energies of hydrogen sorption; raw data for the derivation of sorption-order parameter; modeling of hydrogen sorption kinetics of Pd nanocube-PMMA; hydrogen diffusion and permeability measurements on neat PMMA and Pd nanocube-PMMA plates; and hydrogen gas sensing test setups (PDF)

\section{AUTHOR INFORMATION}

\section{Corresponding Authors}

Kasper Moth-Poulsen - Department of Chemistry and Chemical Engineering, Chalmers University of Technology, 412 96 Göteborg, Sweden; 다이.org/0000-0003-4018-4927; Email: kasper.moth-poulsen@chalmers.se

Christian Müller - Department of Chemistry and Chemical Engineering, Chalmers University of Technology, 41296 Göteborg, Sweden; 이이이.org/0000-0001-7859-7909; Email: christian.muller@chalmers.se

Christoph Langhammer - Department of Physics, Chalmers University of Technology, 41296 Göteborg, Sweden; ○ orcid.org/0000-0003-2180-1379; Email: clangham@ chalmers.se

\section{Authors}

Iwan Darmadi - Department of Physics, Chalmers University of

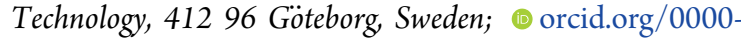
0002-5921-9336

Alicja Stolas - Department of Chemistry and Chemical Engineering, Chalmers University of Technology, 41296 Göteborg, Sweden; 이이.0rid.0000-0002-6736-9553

Ida Östergren - Department of Chemistry and Chemical Engineering, Chalmers University of Technology, 41296 Göteborg, Sweden

Barbara Berke - Department of Physics, Chalmers University of Technology, 41296 Göteborg, Sweden; (ㄱ) orcid.org/00000002-3105-2036

Ferry Anggoro Ardy Nugroho - Department of Physics, Chalmers University of Technology, 41296 Göteborg, Sweden; (i) orcid.org/0000-0001-5571-0454

Matteo Minelli - Department of Civil, Chemical, Environmental and Materials Engineering, University of Bologna, 40131 Bologna, Italy; ○ orcid.org/0000-0003-4662-1526

Sarah Lerch - Department of Chemistry and Chemical Engineering, Chalmers University of Technology, 41296 Göteborg, Sweden; orcid.org/0000-0001-5968-8178

Irem Tanyeli - Department of Physics, Chalmers University of Technology, 41296 Göteborg, Sweden; Insplorion AB, 41390 Göteborg, Sweden; O orcid.org/0000-0002-5433-2524

Anja Lund - Department of Chemistry and Chemical Engineering, Chalmers University of Technology, 41296 Göteborg, Sweden

Olof Andersson - Insplorion AB, 41390 Göteborg, Sweden

Vladimir P. Zhdanov - Department of Physics, Chalmers University of Technology, 41296 Göteborg, Sweden; Boreskov Institute of Catalysis, Russian Academy of Sciences, Novosibirsk 630090, Russia
Marianne Liebi - Department of Physics, Chalmers University of Technology, 41296 Göteborg, Sweden; 이이이.org/00000002-5403-0593

Complete contact information is available at:

https://pubs.acs.org/10.1021/acsanm.0c01907

\section{Author Contributions}

${ }^{\#}$ I.D., A.S., and I.Ö. contributed equally.

\section{Author Contributions}

I.D. executed and analyzed the $\mathrm{H}_{2}$ sensing experiments and the optical characterization of the composite; participated in the composite material planning; made all figures; and assisted during the writing of the manuscript. A.S. synthesized all nanoparticles, executed TEM analysis, participated in the composite material planning, and assisted during the writing of the manuscript. I.O. compounded, melt-pressed, and 3Dprinted all nanocomposite samples; executed the elemental analysis; participated in the composite material planning; and assisted during the writing of the manuscript. B.B. co-executed the scanning-SAXS measurements and the corresponding data analysis. F.A.A.N. participated in the planning, execution, and analysis of $\mathrm{H}_{2}$ sensing experiments. M.M. executed the $\mathrm{H}_{2}$ permeability measurements for the $\mathrm{Pd}$ nanocube-PMMA system and performed the corresponding data analysis. S.L. participated in the nanocrystal synthesis. I.T. executed the $\mathrm{H}_{2}$ sensing experiments with the commercial device. A.L. assisted in the compounding and $3 \mathrm{D}$ printing of nanocomposites. O.A. performed the data analysis from the commercial device. V.P.Z. developed the diffusion front model and corresponding analysis. M.L. co-executed the scanning-SAXS measurements and assisted in the corresponding data analysis. K.M.-P. coordinated the nanoparticle synthesis part of the project. C.M. coordinated the nanocomposite compounding and 3D printing part of the project. C.L. coordinated the sensing part of the work, as well as the project as a whole, and wrote the manuscript.

\section{Notes}

The authors declare the following competing financial interest(s): C.L. is co-founder of Insplorion AB.

All experimental data within the article and its Supplementary Information are available from the corresponding authors upon reasonable request.

\section{ACKNOWLEDGMENTS}

The authors acknowledge financial support from the Swedish Foundation for Strategic Research projects RMA15-0052 and FFL15-0147, and the Knut and Alice Wallenberg Foundation project 2016.0210. The authors also acknowledge the Centre for Cellular Imaging at the University of Gothenburg and the National Microscopy Infrastructure, NMI (VR-RFI 201600968), for providing assistance in microtome sectioning. Finally, the authors thank Manuel Guizar Sicarios at the Swiss Light Source at the Paul Scherrer Institute for his support during the SAXS experiments and Dr. Petri Murto for taking the photographs of the nanocomposite plates. Part of this work was carried out at the Chalmers Materials Analysis Laboratory, CMAL.

\section{REFERENCES}

(1) Anker, J. N.; Hall, W. P.; Lyandres, O.; Shah, N. C.; Zhao, J.; Van Duyne, R. P. Biosensing with Plasmonic Nanosensors. Nat. Mater. 2008, 7, 442-453. 
(2) Larsson, E. M.; Langhammer, C.; Zorić, I.; Kasemo, B. Nanoplasmonic Probes of Catalytic Reactions. Science 2009, 326, 1091-1094.

(3) Syrenova, S.; Wadell, C.; Nugroho, F. A. A.; Gschneidtner, T. A.; Diaz Fernandez, Y. A.; Nalin, G.; Switlik, D.; Westerlund, F.; Antosiewicz, T. J.; Zhdanov, V. P.; Moth-Poulsen, K.; Langhammer, C. Hydride Formation Thermodynamics and Hysteresis in Individual Pd Nanocrystals with Different Size and Shape. Nat. Mater. 2015, 14, $1236-1244$.

(4) Mayer, K. M.; Hafner, J. H. Localized Surface Plasmon Resonance Sensors. Chem. Rev. 2011, 111, 3828-3857.

(5) Tittl, A.; Giessen, H.; Liu, N. Plasmonic Gas and Chemical Sensing. Nanophotonics 2014, 3, 157-180.

(6) Wadell, C.; Syrenova, S.; Langhammer, C. Plasmonic Hydrogen Sensing with Nanostructured Metal Hydrides. ACS Nano 2014, 8, 11925-11940.

(7) Nugroho, F. A. A.; Darmadi, I.; Cusinato, L.; Susarrey-Arce, A.; Schreuders, H.; Bannenberg, L. J.; da Silva Fanta, A. B.; Kadkhodazadeh, S.; Wagner, J. B.; Antosiewicz, T. J.; Hellman, A.; Zhdanov, V. P.; Dam, B.; Langhammer, C. Metal-Polymer Hybrid Nanomaterials for Plasmonic Ultrafast Hydrogen Detection. Nat. Mater. 2019, 18, 489-495.

(8) Hydrogen to the Rescue. Nat. Mater. 2018, 17565. DOI: $10.1038 / \mathrm{s} 41563-018-0129-y$.

(9) Adams, B. D.; Chen, A. The Role of Palladium in a Hydrogen Economy. Mater. Today 2011, 14, 282-289.

(10) Poyli, M. A.; Silkin, V. M.; Chernov, I. P.; Echenique, P. M.; Muiño, R. D.; Aizpurua, J. Multiscale Theoretical Modeling of Plasmonic Sensing of Hydrogen Uptake in Palladium Nanodisks. J. Phys. Chem. Lett. 2012, 3, 2556-2561.

(11) Xia, Y.; Xiong, Y.; Lim, B.; Skrabalak, S. E. Shape-Controlled Synthesis of Metal Nanocrystals: Simple Chemistry Meets Complex Physics? Angew. Chem., Int. Ed. 2009, 48, 60-103.

(12) Kalanur, S. S.; Lee, Y.-A.; Seo, H. Eye-Readable Gasochromic and Optical Hydrogen Gas Sensor Based on CuS-Pd. RSC Adv. 2015, 5, 9028-9034.

(13) Jiang, R.; Qin, F.; Ruan, Q.; Wang, J.; Jin, C. Ultrasensitive Plasmonic Response of Bimetallic $\mathrm{Au} / \mathrm{Pd}$ Nanostructures to Hydrogen. Adv. Funct. Mater. 2014, 24, 7328-7337.

(14) Sacanna, S.; Korpics, M.; Rodriguez, K.; Colón-Meléndez, L.; Kim, S.-H.; Pine, D. J.; Yi, G.-R. Shaping Colloids for Self-Assembly. Nat. Commun. 2013, 4, No. 1688.

(15) Diaz Fernandez, Y. A.; Gschneidtner, T. A.; Wadell, C.; Fornander, L. H.; Lara Avila, S.; Langhammer, C.; Westerlund, F.; Moth-Poulsen, K. The Conquest of Middle-Earth: Combining Topdown and Bottom-up Nanofabrication for Constructing Nanoparticle Based Devices. Nanoscale 2014, 6, 14605-14616.

(16) Zhang, J.; Li, Y.; Zhang, X.; Yang, B. Colloidal Self-Assembly Meets Nanofabrication: From Two-Dimensional Colloidal Crystals to Nanostructure Arrays. Adv. Mater. 2010, 22, 4249-4269.

(17) Darmadi, I.; Nugroho, F. A. A.; Kadkhodazadeh, S.; Wagner, J. B.; Langhammer, C. Rationally Designed PdAuCu Ternary Alloy Nanoparticles for Intrinsically Deactivation-Resistant Ultrafast Plasmonic Hydrogen Sensing. ACS Sens. 2019, 4, 1424-1432.

(18) Palmisano, V.; Weidner, E.; Boon-Brett, L.; Bonato, C.; Harskamp, F.; Moretto, P.; Post, M. B.; Burgess, R.; Rivkin, C.; Buttner, W. J. Selectivity and Resistance to Poisons of Commercial Hydrogen Sensors. Int. J. Hydrogen Energy 2015, 40, 11740-11747.

(19) Clerbaux, C.; Edwards, D. P.; Deeter, M.; Emmons, L.; Lamarque, J. F.; Tie, X. X.; Massie, S. T.; Gille, J. Carbon Monoxide Pollution from Cities and Urban Areas Observed by the Terra/ MOPITT Mission. Geophys. Res. Lett. 2008, 35, No. L03817.

(20) Hong, J.; Lee, S.; Seo, J.; Pyo, S.; Kim, J.; Lee, T. A Highly Sensitive Hydrogen Sensor with Gas Selectivity Using a PMMA Membrane-Coated Pd Nanoparticle/Single-Layer Graphene Hybrid. ACS Appl. Mater. Interfaces 2015, 7, 3554-3561.

(21) Whitelam, S.; Hedges, L. O.; Urban, J. J.; Pint, C. L.; Javey, A.; Bardhan, R. Uncovering the Intrinsic Size Dependence of Hydriding
Phase Transformations in Nanocrystals. Nat. Mater. 2013, 12, 905912.

(22) Baldi, A.; Narayan, T. C.; Koh, A. L.; Dionne, J. A. In Situ Detection of Hydrogen-Induced Phase Transitions in Individual Palladium Nanocrystals. Nat. Mater. 2014, 13, 1143-1148.

(23) Jeon, K. J.; Moon, H. R.; Ruminski, A. M.; Jiang, B.; Kisielowski, C.; Bardhan, R.; Urban, J. J. Air-Stable Magnesium Nanocomposites Provide Rapid and High-Capacity Hydrogen Storage without Using Heavy-Metal Catalysts. Nat. Mater. 2011, 10, 286290.

(24) Langhammer, C.; Kasemo, B.; Zorić, I. Absorption and Scattering of Light by Pt, Pd, Ag, and Au Nanodisks: Absolute Cross Sections and Branching Ratios. J. Chem. Phys. 2007, 126, No. 194702.

(25) Zoríc, I.; Zäch, M.; Kasemo, B.; Langhammer, C. Gold, Platinum, and Aluminum Nanodisk Plasmons: Material Independence, Subradiance, and Damping Mechanisms. ACS Nano 2011, 5, $2535-2546$.

(26) Wadell, C.; Nugroho, F. A. A.; Lidström, E.; Iandolo, B.; Wagner, J. B.; Langhammer, C. Hysteresis-Free Nanoplasmonic PdAu Alloy Hydrogen Sensors. Nano Lett. 2015, 15, 3563-3570.

(27) Chen, P.; Tran, N. T.; Wen, X.; Xiong, Q.; Liedberg, B. Inflection Point of the Localized Surface Plasmon Resonance Peak: A General Method to Improve the Sensitivity. ACS Sens. 2017, 2, 235242 .

(28) Langhammer, C.; Zorić, I.; Larsson, E. M.; Kasemo, B. Localized Surface Plasmons Shed Light on Nanoscale Metal Hydrides. Adv. Mater. 2010, 22, 4628-4633.

(29) Nugroho, F. A. A.; Darmadi, I.; Zhdanov, V. P.; Langhammer C. Universal Scaling and Design Rules of Hydrogen-Induced Optical Properties in Pd and Pd-Alloy Nanoparticles. ACS Nano 2018, 12, 9903-9912.

(30) Grönbeck, H.; Zhdanov, V. P. Effect of Lattice Strain on Hydrogen Diffusion in Pd: A Density Functional Theory Study. Phys. Rev. B 2011, 84, No. 052301.

(31) Minelli, M.; Sarti, G. C. Elementary Prediction of Gas Permeability in Glassy Polymers. J. Membr. Sci. 2017, 521, 73-83.

(32) Niu, W.; Li, Z. Y.; Shi, L.; Liu, X.; Li, H.; Han, S.; Chen, J.; Xu, G. Seed-Mediated Growth of Nearly Monodisperse Palladium Nanocubes with Controllable Sizes. Cryst. Growth Des. 2008, 8, 4440-4444.

(33) Bunk, O.; Bech, M.; Jensen, T. H.; Feidenhans'L, R.; Binderup, T.; Menzel, A.; Pfeiffer, F. Multimodal X-Ray Scatter Imaging. New J. Phys. 2009, 11, No. 123016. 